\title{
Lavender Fragrance Essential Oil and the Quality of Sleep in Postpartum Women
}

\author{
Mahnaz Keshavarz Afshar ${ }^{1}$; Zahra Behboodi Moghadam ${ }^{2,}$; Ziba Taghizadeh ${ }^{2}$; Reza \\ Bekhradi ${ }^{3}$; Ali Montazeri ${ }^{4}$; Pouran Mokhtari ${ }^{1}$ \\ ${ }^{1}$ Department of Midwifery, School of Nursing and Midwifery, Zanjan University of Medical Sciences, Zanjan, IR Iran \\ ${ }_{3}^{2}$ Department of Reproductive Health, Nursing and Midwifery Faculty, Tehran University of Medical Sciences, Tehran, IR Iran \\ ${ }_{4}^{3}$ Research and Development Unit, Barij Essence Company, Kashan, IR Iran \\ ${ }^{4}$ Mental Health Research Group, Health Metrics Research Center, Iranian Institute for Health Sciences Research, ACECR, Tehran, IR Iran \\ *Corresponding Author: Zahra Behboodi Moghadam, Department of Reproductive Health, Nursing and Midwifery Faculty, Tehran University of Medical Sciences, Tehran, IR Iran. \\ Tel:+98-9122494201, Fax+98-2166927171, E-mail: Behboodi@tums.ac.ir
}

Received: December 10, 2014; Revised: December 28, 2014; Accepted: January 11, 2015

\begin{abstract}
Background: Labor and delivery is a stressful stage for mothers. During these periods, sleep-related disorders have been reported. The problems of inadequate sleep include decrease in concentration, judgment, difficulty in performing daily activities, and an increase in irritability. Even the effects of moderate sleep loss on life and health quality can be similar to sleep deprivation. some research aggravated by aromatherapy on sleep quality in different periods of life so might be useful for the improve of sleep quality in postpartum women. Objectives: This study aimed to determine the effect of aromatherapy on the quality of sleep in postpartum women. The sample was recruited from medical health centers of Zanjan University of Medical Sciences.

Patients and Methods: This study was a randomized clinical trial with the control group. A total of 158 mothers in postpartum period (with certain inclusion criteria) were enrolled in the study and assigned randomly to two groups of control and intervention. Lavender fragrance (made by Barij Essence Pharmaceutical Co.) was used by participants in the intervention group nightly before sleeping. The fragrance was dropped on cotton balls, which were placed on a cylindrical container at mothers' disposal. Keeping the container at a projected distance of $20 \mathrm{~cm}$, the participants inhaled 10 deep breaths and then the container was placed beside their pillow until morning. This procedure was done 4 times a week for 8 weeks. For the control group, the sameintervention was done with the placebo. The instrument for collecting data was Pittsburgh sleep quality index, which was completed at the baseline, fourth, and eighth weeks after the intervention. Data were analyzed using independent t test and repeated measures analysis of variance calculated by SPSS16.

Results: Before the intervention, there were no significant differences between mothers in two groups $(\mathrm{P}>0.05)$. After 8 weeks follow up, a significant improvement appeared in mothers' sleep quality in the intervention group. Aromatherapy increased sleep quality mean score $( \pm S D)$ from $8.2911( \pm 2.1192)$ to $6.7975( \pm 2.3663)(\mathrm{P}<0.05)$, but in the control group sleep quality mean score $( \pm S D)$ changes from $8.4557( \pm$ $2.3027)$ to $7.5696( \pm 1.1464)(\mathrm{P}>0.05)$. Comparing sleep quality between control and intervention groups after 8 weeks from the beginning of the intervention indicated that aromatherapy was effective in the improvement of mothers' sleep quality $(\mathrm{P}<0.05)$.

Conclusions: Considering the effects of aromatherapy on the improvement of mother's sleep quality during postpartum period, aromatherapy has been suggested as a non-pharmacological method for the improvement of the maternal health.
\end{abstract}

Keywords: Sleep Disorders; Aromatherapy; Lavandula; Postpartum Period

\section{Background}

Labor and delivery is a stressful stage for mothers. This stage entails a change in their life style (1). Pregnancy and postpartum are two important stages for women and their families (2). During these periods, sleep-related disorders have been reported (3). Sleep is an important process in human life. The problems of inadequate sleep include decrease in concentration, judgment, difficulty in performing daily activities, and an increase in irritability (4). Research shows that inadequate sleep affects hemostatic and nerve functions, as well as mood. Even the effects of moderate sleep loss on life and health quality can be similar to sleep deprivation. Many medical practitioners and patients are faced with such prob- lems (5). Most mothers experience changes in their quality of sleep during postpartum period. According to the researches, changes in sleep duration and its patterns have normally been reported. Sleep problems are related to pain, wound discomfort, lactation, or uterine contractions (6). Mothers in postpartum period normally experience $20 \%$ increase in their wakefulness during night (7). According to the National Sleep Foundation survey in $2007,67 \%$ of women in the postpartum period reported disturbed sleep cycles (8).

In general, changes in sleep patterns during the first year after delivery is the distinctive feature of this period, which affects health and functions of mothers and 
creates a lot of problems not only for them but also for their families (9). One of the most important side effects of inadequate quality of sleep is the incident and severity of postpartum depression $(3,10)$. Another adverse side effect of impaired sleep patterns is the cessation of breastfeeding and decreased mother-infant attachment (11). Sleep deprivation can even decrease the growth of infant (12), and reduce mothers' immune functioning, metabolism, mood, and performance (13).

For many reasons, intervention and studies of sleeping patterns after delivery are very important and sensitive for a nascent family at this time. However, studying sleep characteristics is a difficult and complicated concept, as in this period, mothers and their families try to incorporate a new member into their family (14). What mothers could do regarding the quality of sleep, its disturbance, and frequency at this crucial time is still being investigated. Physical and mental health of mothers, newborn outcomes, and maternal-infant relationship are affected by fully understanding and correct recognition of sleep during pregnancy and postpartum (3). Midwives usually train mothers to reduce fatigue and improve sleep, which most of these trainings are generally not evidence-based (14). Drug therapy is another way to improve sleep quality; however, it has many side effects and not the best way to improve sleep quality (15). Because of the mothers' concerns about the side effects of drugs for themselves or for their nursing baby, they seek alternative and complementary methods for treating their symptoms. Therefore, the use of alternative medicine is better for patients (15). Alternative therapies for the treatment of various physical and mental distresses are available for young women (16).

One of the treatment methods, which has expanded in recent years is aromatherapy. This treatment is the second alternative treatment method, which uses essential oil extracted from aromatic plants for the treatment. Lavender (Lavandula angustifolia), which belongs to green mint family and aromatic plants is one of the most used aromatic plants in aromatherapy (15). Several studies have been conducted on the effects of aromatherapy in sleep-related disorders. For example, in a study conducted by Chien et al. the overall score of quality of sleep has been improved in the group who did inhale lavender aroma (17). According to the research conducted by Lee et al. who studied the effects of Lavender oil aromatherapy on insomnia in Korean female students, it was found that this treatment significantly reduces insomnia (18). Also, the research of Moeini et al. showed that lavender aromatherapy improved sleep quality in patients in CCU (15). In another study, Arzi et al. showed that the scent of lavender only improved the repeated wakening and had no other effects (19). However, there have been a few clinical trials that studied the improvement of mothers' sleep quality during postpartum period. For example, in a study conducted by Lee et al. with the purpose of investigating the effects of aro- matherapy on sleep quality and fatigue in postpartum mothers, the results showed that inhaled aromatherapy has no significant effects on sleep quality with regard to sleep duration, the numbers of waking up at nights, and sleep satisfaction (11).

Given the importance of sleep quality in postpartum period, its adverse effects on the quality of life and ability of midwives to prescribe and use complementary medicine and considering the lack of studies in Iran on the effectiveness of aromatherapy (on sleep quality in this period of life), we decided to assess the effects of aromatherapy on sleep quality in postpartum period.

\section{Objectives}

This study aimed to determine the effect of aromatherapy on the quality of sleep in postpartum women. The sample was recruited from medical health centers of Zanjan University of Medical Sciences. This study was carried out with the hypothesis that the mean score of quality of sleep would be better in postpartum women in aromatherapy group compared to control group.

\section{Patients and Methods}

This study was a randomized clinical trial with a control group, in which the data were collected from both groups before and after the intervention. The study population consisted of all women who have recently given birth and covered by health-care referral governmental centers affiliated to Zanjan University of Medical Sciences. This study was registered in Iranian Registry of Clinical Trials (registration number of IRCT201211179463N8) and approved by the Ethics Committee of Tehran University of Medical Sciences (No.130/904/D/92).

The sampling began in December 19, 2013 and lasted for 6 months. The sample size was calculated according to the previous articles $(15,20)$ and by the advice of the statistics professor using OpenEpi (www.OpenEpi. com) software (21). The number of individuals in each group was calculated as 61 . Because of the availability of samples and willingness of individuals to participate in the study, 79 subjects were enrolled in each group. Out of 4 health centers in Zanjan that conducted hypothyroidism and phenylketonuria screening tests, 2 of them have been chosen randomly and sampling of intervention and control groups began at those centers. The reason for choosing centers that provide neonatal screening for sampling was the attendance of the mothers in first few days after delivery as well as future referrals for postpartum care, which provided an easy access to samples for the research. Participants satisfied inclusion criteria were enrolled after completing an informed consent form. Inclusion criteria included primiparous women, uncomplicated vaginal delivery, a minimum literacy tips, aged 18 - 35 years, absence of acute or chronic physical and mental illness, being exclusive breast-feeders of their infants, having healthy 
baby without complications, and sleep disorders (The Pittsburgh Sleep Quality Index score of 5 or higher). Exclusion criteria were having chronic diseases (diabetes), using certain drugs such as hypnotics or sedatives, having a history of allergy to herbal remedies, and suffering from depression. Randomization was performed by simple allocation. Sampling continued until 79 patients were allocated in each group. Then, the researcher followed the participants with phone calls and text messages and reminded them about the intervention so that nobody dropped out of the study.

The aromatherapy blend used in this study consisted of $10 \%$ lavender essential oil of sesame carrier oil and placebo was just sesame carrier oil, which was produced by Barij Essence Pharmaceutical Co. in Iran under Batch No 9208051. The intervention consisted of inhaling the aroma of lavender essential oil, by the intervention group used at night and before sleeping. They dropped 4 drops of essential oil on a cotton ball, placed on a cylindrical container at their disposal and with a projected distance of $20 \mathrm{~cm}$. They inhaled 10 deep breaths and then placed the container beside their pillow until morning. The control group was also treated in the same manner except that a placebo was inhaled by the participants. All participants were asked to inhale the lavender essential oil during bed time until the next morning and preferably for 4 consecutive days per week for 8 weeks. To ensure its proper execution, a check list was provided for the participants, and each week, the researcher reminded the participants of the intervention with a phone call or short text message. Furthermore, the contact number of the researcher was provided for the participants. If for any reason, subjects did not complete the aromatherapy for 4 times a week, they would be excluded from the study.

In this study, the tools that have been used by the researcher for demographic data collection comprised a questionnaire survey of environmental confounding factors affecting sleep, Pittsburgh sleep quality index, and the Edinburgh Postnatal Depression Scale as well as aromatherapy and registration forms that were completed by mothers. Pittsburgh sleep quality index is an international standardized tool that has been validated in numerous studies $(15,20)$. Test-retest reliability and content validity of its Iranian version have been reviewed and approved in two studies done by Hossein Abadi et al. (4) and Malekzadegan et al. (22) whose results showed the specificity of $87 \%$ with a sensitivity of $90 \%$. Test-retest reliability has been determined in the study of Malekzadegan et al. (22) and Cronbach $\alpha$ was evaluated at $88 \%$ in the study of Hossein Abadi et al. (4, 20, 22, 23). Pittsburgh Sleep Quality Index consisted of 19 questions that evaluate the quality of sleep in the past month and measures 7 dimensions (24). The total score of this index ranges from 0 to 21, which a total score of 5 or more denotes poor sleep quality and shows that the individual has some problems in 2 or more dimensions (24). This scale was completed by the researcher in the meetings with the mothers at the health centers 3 - 5 days after birth, then at the end of the first four weeks and finally at the end of the second four weeks after labor. Because of the poor quality of sleep in cases of postpartum depression in mothers (10), and its effect as a confounding factor, Edinburgh Postnatal Depression Scale has been performed by the researcher in health centers at the beginning of the study and before the intervention. In case of scoring 10 or higher, the samples were excluded from the study with a diagnosis of postpartum depression. Edinburgh Postnatal Depression Scale is a universal standardized tool that its reliability and validity have been confirmed by Boyed and colleagues (20). Confounding factors have been also examined by a researcher form that has been performed before the intervention.

\subsection{Statistical Analysis}

Descriptive statistics, including means and standard deviations were calculated for all variables. Independent t test, Chi-square, and repeated measure test were used to compare means of the impact of lavandula aromatherapy and placebo on the sleep quality between two groups (case and control). The data were analyzed using version 16 of SPSS software. The characteristics of the participants are presented as mean (SD), or number (percentage). Differences between variables were determined by independent $t$ test and repeated measure test. P value $<0.05$ was considered as significant (Figure 1).

\section{Results}

Demographic characteristics of study subjects and their husbands are shown in Table 1 . Mothers in both groups were similar in all demographic characteristics ( $P$ $>0.05)$. In terms of individual-environmental confounding factors affecting sleep disorders such as lighting a bedroom, existence of noise, consuming tea and coffee, consuming caffeinated soft drinks, herbal remedies, complaining of pain, general sleep conditions of infants, and assistance in infants care in two groups showed no significant differences $(P>0.05)$. Table 2 shows the mean and standard deviation of sleep quality index at the beginning of the study, at the end of the four weeks, and eight weeks after intervention in two groups. As a result, the mean score of sleep quality at baseline in aromatherapy was ( $8.28 \pm 2.11)$ and in placebo group was (8.45 \pm 2.30 ) and before the intervention there was no significant difference between the two groups in mean score of quality of sleep $(\mathrm{P}>0.05)$. Four weeks after the intervention, the result showed the mean of quality of sleep in aromatherapy group was $(7.59 \pm 2.53)$ and placebo group was (8.06 \pm 2.20$)$, and finally, the average of the quality of sleep after eight weeks in the intervention group was $(6.79 \pm 2.36)$ and in the placebo group was $(7.56 \pm 2.14)$. Analysis of the data revealed that despite the improvements in sleep quality after 4 weeks of intervention, 


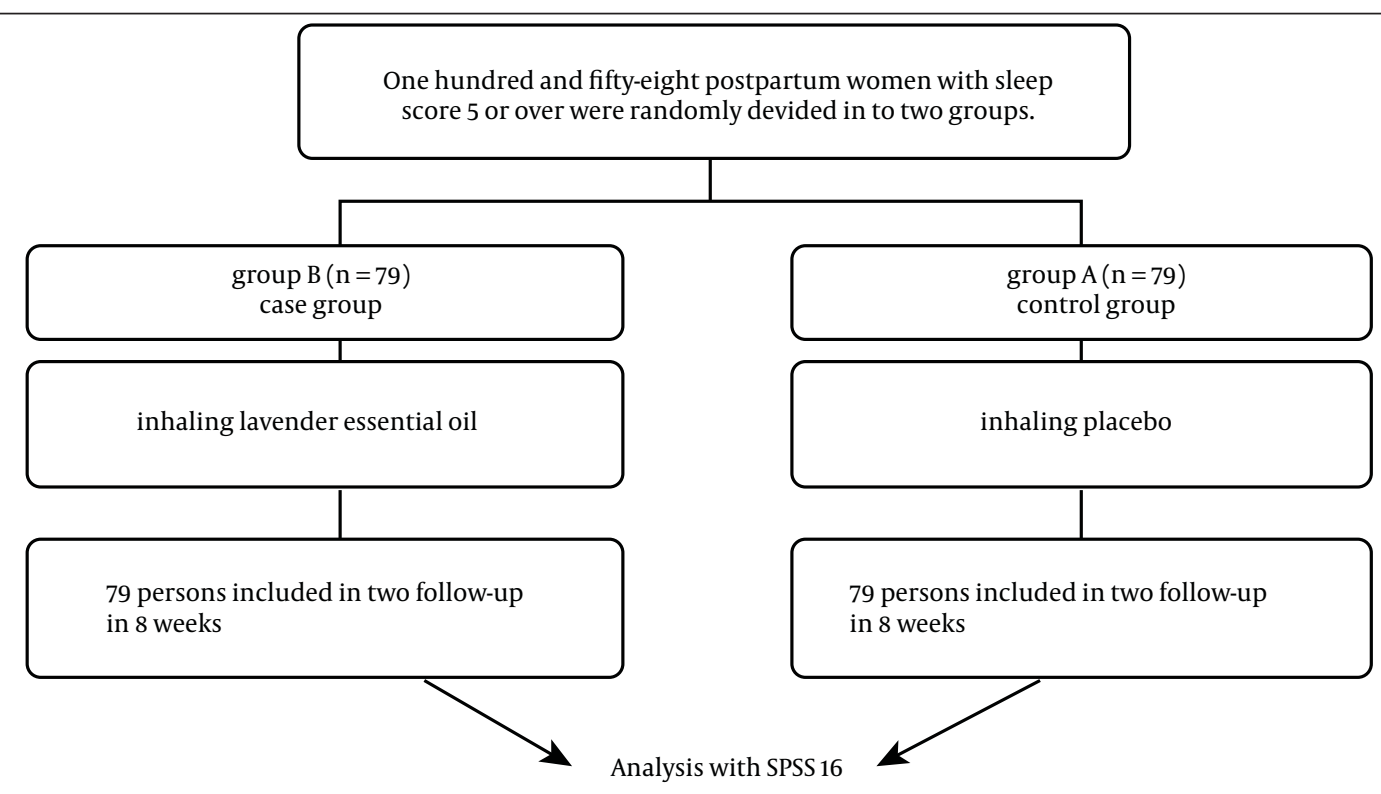

Figure 1. Study Method Design

\begin{tabular}{|c|c|c|c|}
\hline \multirow[t]{2}{*}{ Variables } & \multicolumn{2}{|c|}{ Group } & \multirow[b]{2}{*}{ Independent t test and Chi-square Test } \\
\hline & Case & Control & \\
\hline Women's mean age, $y$ & $28.06 \pm 4.105$ & $28.14 \pm 4.128$ & P Value $=0.908$ \\
\hline Husbands' mean age, $y$ & $31.75 \pm 5.06$ & $32.12 \pm 5.05$ & PValue $=0.649$ \\
\hline Women's degree of education & & & P Value $=0.726$ \\
\hline Primary School & $3(3.8)$ & $2(2.5)$ & \\
\hline High school & $3(3.8)$ & $6(7.6)$ & \\
\hline Diploma & $24(30.4)$ & $25(31.6)$ & \\
\hline University & $49(62)$ & $46(58.2)$ & \\
\hline Husbands' degree of education & & & P Value $=0.854$ \\
\hline Primary school & $3(3.8)$ & $2(2.5)$ & \\
\hline High school & $6(7.6)$ & $6(7.6)$ & \\
\hline Diploma & $49(52)$ & $44(55.7)$ & \\
\hline University & $21(26.6)$ & $27(34.2)$ & \\
\hline Womens' job & & & P Value $=0.869$ \\
\hline Housewife & $58(73.2)$ & $59(72.7)$ & \\
\hline Employee & $12(15.3)$ & $14(17.7)$ & \\
\hline Student & $6(7.7)$ & $5(6.3)$ & \\
\hline Self-employed & $3(3.8)$ & $1(1.3)$ & \\
\hline Husbands' job & & & P Value $=0.352$ \\
\hline Employee & $28(35.4)$ & $23(29.1)$ & \\
\hline Worker & $15(19)$ & $10(12.7)$ & \\
\hline Self-employed & $33(41.8)$ & $41(51.9)$ & \\
\hline other & $3(3.8)$ & $5(6.3)$ & \\
\hline The income from the perspective of women & & & P Value $=0.897$ \\
\hline Sufficient & $27(34.2)$ & $28(35.4)$ & \\
\hline Nearly Sufficient & $49(62)$ & $49(62)$ & \\
\hline Insufficient & $3(3.8)$ & $2(2.5)$ & \\
\hline
\end{tabular}

\footnotetext{
${ }^{\mathrm{a}}$ Data are presented as No. (\%) or Mean \pm SD.
} 
Keshavarz Afshar M et al.

there were no significant differences between the intervention and control groups $(P>0.05)$. However, eight weeks after intervention, a significant difference was observed between two groups $(\mathrm{P}<0.05)$. To examine differences in sleep quality means between mothers in both groups over time, repeated measures tests have been performed. Before performing this analysis, assumptions, including homogeneity of variances and normal response variables were examined, which confirmed the establishment of the assumptions. By considering time and intervention concurrency at the end of eight weeks compared to the pre-intervention condition, aromatherapy significantly increased sleep quality from $(8.28 \pm 2.11)$ to $(6.79 \pm 2.36)(\mathrm{P}>0.05)$. While at the end of eight weeks (compared to the pre-intervention conditions), no significant change was observed in placebo group in comparison to aroma group: $8.45 \pm 2.30$ to $7.56 \pm 2.14(\mathrm{P}<0.05)$ (Table 3, Figures 2 and 3).

Table 2. Comparison of the Quality of Sleep in Postpartum Women Between Case and Control Groups Before and After the Intervention

\begin{tabular}{lcc}
\hline Variable Intervention & Mean \pm SD & P Value $^{\mathrm{a}}$ \\
\hline Before intervention & & 0.64 \\
Case & $8.2911 \pm 2.11922$ & 0.216 \\
\hline Control & $8.4557 \pm 2.30272$ & \\
$\mathbf{4}$ weeks after intervention & & 0.033 \\
\hline Case & $7.5949 \pm 2.52941$ & \\
\hline Control & $8.0633 \pm 2.20339$ & \\
\hline $\mathbf{8}$ weeks after intervention & & \\
Case & $6.7975 \pm 2.36632$ & \\
\hline Control & $7.5696 \pm 1.14646$ & \\
\hline
\end{tabular}

$\mathrm{a}$ Independent $\mathrm{t}$ test.

Table 3. Comparison of the Quality of Sleep Between Postpartum Women in Control and Case Groups After the Intervention ${ }^{\mathrm{a}, \mathrm{b}}$

\begin{tabular}{lcccc}
\hline Variable & Before Intervention & 4 Weeks After Intervention & 8 Weeks After Intervention & PValue $^{\mathrm{C}}$ \\
\hline Quality of Sleep & & & & 0.002 \\
Case & $8.2911 \pm 2.1192$ & $7.5949 \pm 2.5294$ & $6.7975 \pm 2.3663$ & \\
Control & $8.4557 \pm 2.3027$ & $8.0633 \pm 2.2033$ & $7.5696 \pm 1.1464$ & \\
\hline
\end{tabular}

a Data are presented as Mean \pm SD.

$\mathrm{b}$ Repeated measure test.

${ }^{\mathrm{C}} \mathrm{P}$ value $<0.05$ were considered as significant.

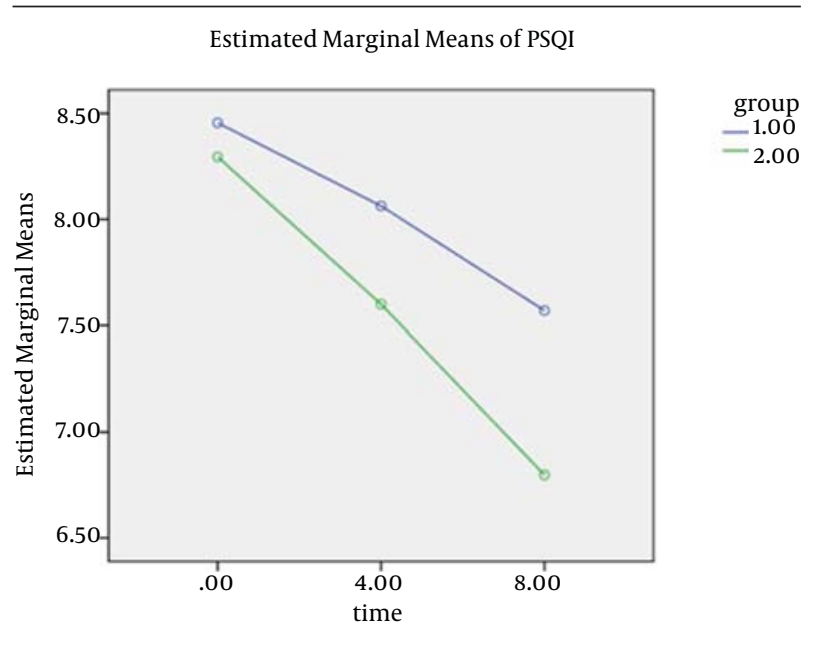

Figure 2. Estimated Marginal Mean of Pittsburgh Sleep Quality Index

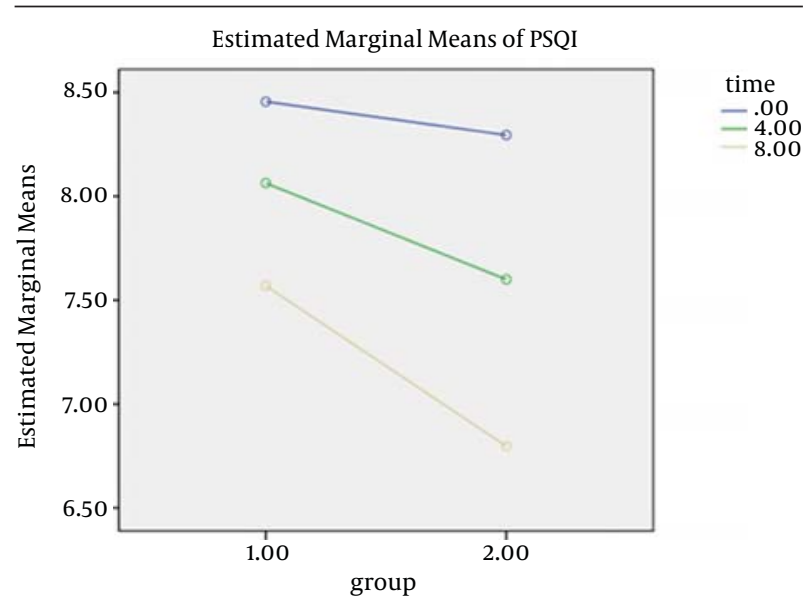

Figure 3. Estimated Marginal Mean of Pittsburgh Sleep Quality Index 


\section{Discussion}

The present study was conducted with the purpose of investigating the effects of aromatherapy on sleep quality of the women during their postpartum period. Comparing two groups of intervention and control showed the effectiveness of aromatherapy on mothers' sleep quality. Inhaling this aroma affected some aspects of sleep quality, including sleep latency and duration, while it had no statistically significant impact on other aspects $(\mathrm{P}<0.05)$.

These findings are consistent with the results of Chien et al. (17) study about the effects of lavender essential oil on autonomic nervous system of middle-aged women suffering from insomnia. The total score of sleep quality after aromatherapy with lavender essential oil reduced after the intervention, but there was no change in sleep quality score in the control group. The results from a study conducted by Hirokawa et al. (23) showed that lavender essential oil improves sleepiness during wakefulness. Also in a study conducted by Moeini et al. (15) the total score of sleep quality in those who did inhale lavender essential oil decreased while in the control group there was no change in sleep quality score after the same period of time. Another study by Field and colleagues (2008) with the purpose of studying the effects of aromatherapy in reducing infants' stress and crying as well as enhancing their sleep showed the improvement of sleep quality after aromatherapy with lavender oil (25). Based on a study done by Lee about the effects of aromatherapy on the quality of sleep and fatigue level of the mothers in the postpartum period, inhaled aromatherapy had no effect on the duration of sleep, frequency of night waking, and sleep satisfaction (11). These different results might be due to the following reasons:

1) mothers in this study were enrolled with adequate sleep quality.

2) duration of aromatherapy was short

3) questionnaire used in the study was a self-designed questionnaire; moreover

4) PSQL, RCSQ and HCAMQ standardized questionnaire that has been used in the previous studies, were not used in this particular study.

Today, aromatherapy has been considered as an alternative medicine approach with a positive impact and tested in various researches however, the mechanism of its effects on sleep quality and its patterns are poorly understood. Nevertheless, in principle the sense of smell plays an important role in overall health as well as physical and mental relaxation and the brain responds emotionally to aroma (24). In the postpartum period, because of the conditions and problems such as pain, sudden hormonal changes in mothers and taking care of the baby, using aromatherapy seems to be beneficial as a convenient and easily accessible method for improving sleep quality of mothers, which results in the infant and family health too. Despite several studies in the field of aromatherapy on sleep quality in different periods of life and under different health conditions, a few studies have investigated the effects of aromatherapy on the sleep quality in women during the critical postpartum period to generalize the results. Therefore, this field of investigation requires more research. The strong points of this paper are the careful and continuous follow-up in addition to use of complementary medicine that has no known compilations. The weak point of this study relates to the factors that affect the quality of sleep and were out of our control. This study is a clinical trial that examined the effects of aromatherapy on sleep quality in women during postpartum period. Lavender aromatherapy improves the quality of sleep in postpartum women.

\section{Acknowledgements}

We would like to thank the participants in this research, all people in charge in Tehran University of Medical Sciences, Zanjan University of Medical Sciences and Barij Essence Company, for their assistance and cooperation.

\section{Funding/Support}

This study was financially supported by Tehran University of Medical Sciences.

\section{Authors' Contributions}

Mahnaz Keshavarz Afshar: Data collection, as well as design and writing the manuscript, Zahra Behboodi Moghadam: Supervisor, design and writing the manuscript, Pouran Mokhtari and Ziba Taghizadeh: Counselor, Ali Montazeri: Data analysis, Reza Bekhradi: Material providing.

\section{Financial Disclosure}

This project has been approved and financially supported by Tehran University of Medical Sciences. The interventional drug and placebo was manufactured by the support of Barij Essence Company.

\section{References}

1. Li CY, Chen SC, Li CY, Gau ML, Huang CM. Randomised controlled trial of the effectiveness of using foot reflexology to improve quality of sleep amongst Taiwanese postpartum women. Midwifery. 2011;27(2):181-6.

2. Imura M, Misao H, Ushijima H. The psychological effects of aromatherapy-massage in healthy postpartum mothers. J Midwifery Womens Health. 2006;51(2):e21-7.

3. Okun ML. Sleep in Pregnancy and the Postpartum. Encyclopedia Sleep. 2013:674-9.

4. Reza H, Kian N, Pouresmail Z, Masood K, Sadat Seyed Bagher M, Cheraghi MA. The effect of acupressure on quality of sleep in Iranian elderly nursing home residents. Complement Ther Clin Pract. 2010;16(2):81-5.

5. Fismer KL, Pilkington K. Lavender and sleep: A systematic review of the evidence. Eur Integr Med. 2012;4(4):e436-e47.

6. Ko YL, Lee HJ. Randomised controlled trial of the effectiveness of using back massage to improve sleep quality among Taiwanese insomnia postpartumwomen. Midwifery. 2014;30(1):60-4.

7. Goyal D, Gay CL, Lee KA. Patterns of sleep disruption and de- 
pressive symptoms in new mothers. J Perinat Neonatal Nurs. 2007;21(2):123-9.

8. National Sleep Foundation.. Sleep in America Poll. Washington, DC: National Sleep Foundation; 2007. Available from: http:// sleepfoundation.org/.

9. Dennis CL, Ross L. Relationships among infant sleep patterns, maternal fatigue, and development of depressive symptomatology. Birth. 2005;32(3):187-93.

10. Posmontier B. Sleep quality in women with and without postpartum depression.J Obstet Gynecol Neonatal Nurs. 2008;37(6):722-35.

11. Lee SH. Effects of aroma inhalation on fatigue and sleep quality of postpartum mothers. Women Health Nurs . 2004;10(3):235-43.

12. Gunderson EP, Rifas-Shiman SL, Oken E, Rich-Edwards JW, Kleinman KP, Taveras EM, et al. Association of fewer hours of sleep at 6 months postpartum with substantial weight retention at 1 year postpartum. Am JEpidemiol. 2008;167(2):178-87.

13. Signal TL, Gander PH, Sangalli MR, Travier N, Firestone RT, Tuohy JF. Sleep duration and quality in healthy nulliparous and multiparous women across pregnancy and post-partum. Aust N Z J Obstet Gynaecol. 2007;47(1):16-22.

14. Rychnovsky J, Hunter LP. The relationship between sleep characteristics and fatigue in healthy postpartum women. Womens Health Issues. 2009;19(1):38-44.

15. Moeini M, Khadibi M, Bekhradi R, Mahmoudian SA, Nazari F. Effect of aromatherapy on the quality of sleep in ischemic heart disease patients hospitalized in intensive care units of heart hospitals of the Isfahan University of Medical Sciences. Iran J Nurs Midwifery Res. 2010;15(4):234-9.

16. Conrad P, Adams C. The effects of clinical aromatherapy for anxiety and depression in the high risk postpartum woman - a pilot study. Complement Ther Clin Pract. 2012;18(3):164-8.

17. Chien LW, Cheng SL, Liu CF. The effect of lavender aromatherapy on autonomic nervous system in midlife women with insomnia. Evid Based Complement Alternat Med. 2012;2012:740813.

18. Lee IS, Lee GJ. [Effects of lavender aromatherapy on insomnia and depression in women college students]. Taehan Kanho Hakhoe Chi. 2006;36(1):136-43.

19. Arzi A, Sela L, Green A, Givaty G, Dagan Y, Sobel N. The influence of odorants on respiratory patterns in sleep. Chem Senses. 2010;35(1):31-40.

20. Jahdi F, Behboodi Moghadam Z, Hagani H. The impact of Health Behavior Education on the Sleep Quality in the Pregnant Women with sleep disorder: A Randomized Control Trial 2011-2012.Int Res J Applied Basic Sci. 2013;7(12):921-8.

21. Sullivan KM, Dean A, Soe MM. OpenEpi: a web-based epidemiologic and statistical calculator for public health. Public Health Rep. 2009;124(3):471-4.

22. Malekzadegan A, Moradkhani M, Ashaeri H, Haghani H. The effects of Relaxation exercise training on the sleep disorders in the third trimester of pregnancy, pregnant women referred to health centers in Zanjan. Sch Nurs Midwifery Iran Univ Med Sci Iran. 2005;1385.

23. Hirokawa K, Nishimoto T, Taniguchi T. Effects of lavender aroma on sleep quality in healthy Japanese students. Percept Mot Skills. 2012;114(1):111-22.

24. Pillitteri A. Maternal \& child health nursing: care of the childbearing and childrearing famil.: Lippincott Williams and Wilkins; 2010.

25. Field T, Field T, Cullen C, Largie S, Diego M, Schanberg S, et al. Lavender bath oil reduces stress and crying and enhances sleep in very young infants. Early Hum Dev. 2008;84(6):399-401. 